rise to epidemics. Successful epidemic strains may require special properties, and it might have been hoped that such strains were unreceptive to transfer of resistance and that multiresistant strains might hence be less highly transmissible and more amenable to containment by modest hygienic measures.

Any such comfortable thoughts are now swept aside by the emergence of epidemic typhoid in Mexico due to a strain possessing transferable chloramphenicol resistance. Widespread use of chloramphenicol and opportunities for transmission of the organism have evidently provided there just the conditions necessary to allow the dominance of multiresistant strains. Added to this, air travel has allowed these disagreeable organisms to be imported into the U.S.A. and, as Dr. E. S. Anderson and Mr. H. R. Smith report at page 329 of the B.M.f. this week, into Britain. While there is doubtless room for improvement in our hygienic practices and use of antibiotics, there are, as Anderson and Smith say, unlikely to be great opportunities for epidemic spread of these organisms here. But their report is an important reminder that these organisms present us with therapeutic problems, serves as a warning of the need to take scrupulous care in the prescribing of antibiotics, and should encourage us to examine prescribing practices in other parts of the world now drawn so close to us.

1 British Medical fournal, 1965, 1, 1325.

2 Anderson, E. S., British Medical fournal, 1968, 3, 333.

\section{Antepartum Pulmonary Embolism}

At the eighty-second annual meeting of the American Association of Obstetricians and Gynaecologists antepartum pulmonary embolism due to blood clot was discussed. The debate was stimulated by S. R. Henderson, C. J. Lund, and W. T. Creasman, ${ }^{1}$ who analysed the frequency and number of deaths from thromboembolic disease among female patients aged 9-50 in hospital between 1959 and 1970. There were 72 cases in the obstetric service compared with 138 in the medical and surgical units in their hospital.

During the 12-year period of study the incidence of thromboembolism was 2.4 per thousand deliveries and thus similar to the figures in other reported series. The accepted range is 1-5 cases per thousand deliveries. However, in their series the disease during pregnancy was almost equally divided between the antepartum and postpartum periods, which is different from most other series. Of particular interest were the 20 cases of antepartum pulmonary embolism (with two maternal deaths) compared with 14 cases of antepartum deep-vein thrombosis. Ten of the emboli occurred in the first trimester, nine in the last trimester, and only one in the middle trimester.

One of the largest series of deaths from pulmonary embolism during pregnancy analysed over recent years have been those reported on by the Ministry of Health. ${ }^{2}$ So far they have not shown such a striking incidence of fatal antepartum embolism in the first trimester, even when allowance is made for those included under abortion as the prime cause of death. For the 15-year period published to date the overall incidence of fatal antepartum embolism was $17 \%$, though for the last two reports, 1961-3 and 1964-6 inclusive, the incidence in each was $25 \%$.
Henderson and colleagues ${ }^{1}$ acknowledge the limits of their detailed retrospective study of 20 cases of antepartum embolism, in all of which there were sound clinical and laboratory data to confirm the diagnosis. The main predisposing factors were age, vascular disease, previous thromboembolism, and obesity. No reference was made to the ABO grouping, which is now recognized ${ }^{3}$ as an associated factor.

The diagnosis of deep-vein thrombosis is generally more difficult in pregnancy than at other times because the pelvic veins are often involved. Furthermore, recent techniques, ${ }^{3-6}$ in particular with iodine-125-labelled fibrinogen, for the detection of deep-vein thrombosis are not usually performed during pregnancy.

The symptoms and signs of embolism are similar in the pregnant and non-pregnant patient. The commonest symptoms are pain and dyspnoea; in addition tachycardia (over $120 / \mathrm{min}$ ) and tachypnoea may be noted. These and cyanosis, if present, result from a combination of systemic hypotension and pulmonary hypertension, but both symptoms and signs vary in accordance with the size and the site of the embolus. The clinical signs on auscultation of the chest are also variable. A chest $x$-ray, though it will not diagnose an embolism with certainty, is a most useful ancillary investigation; lung scans and pulmonary arteriography may help, but all take time when the initiation of treatment is a matter of urgency. The electrocardiogram is not helpful, though it undoubtedly does show changes.

The presence of the symptoms mentioned above in a patient who has a deep-vein thrombosis is diagnostic of embolism. With regard to the diagnosis of antepartum pulmonary embolism: it should always be suspected. Because of the potentially fatal outcome, any pain in the leg or chest in pregnancy should be considered to be due to deepvein thrombosis or pulmonary embolism until proved otherwise, and it should be treated as such until the diagivivis is confirmed. ${ }^{7}$

The diagnosis of pulmonary embolism having been made or suspected, treatment should be started urgently to avoid risk of a fatal outcome. In the initial stage the best drug to give is intravenous heparin, but pain and anxiety may need to be allayed with analgesics such as the opiates. Controversy exists on the best way of prescribing the heparin, but suggestions have included (1) 10,000 units intravenously 6-hourly, or (2) heparin 10,000 units 4-hourly for 12-24 hours and thereafter 6-hourly, or (3) a heparin drip in which 40,000 units is given over 24 hours and thereafter reduced to 20,000 units per 24 hours to avoid overdosage. Each of these regimens has its merits. In general the intermittent therapy requires little control (especially the 6hourly regimen), but the continuous method does. In the doses suggested there is at present no evidence that heparin reaches the fetus. The heparin should be continued for 10 14 days (or even longer) according to the patient's progress. After this (or earlier if preferred) the obstetrician has to decide whether to continue intermittent therapy through an indwelling needle or change over to oral anticoagulants, which present a risk to the fetus because they cross the placental membrane. If the embolism occurs early in pregnancy this risk may have to be taken. If oral anticoagulants are given the patient should be switched to heparin before the induction or onset of labour, because it has a rapid antidote whereas the oral anticoagulants do not. Prophylactic therapy should preferably be given for at least six to eight weeks postpartum to avoid the danger of pulmonary hypertension and its inevitable sequelae.

The precise roles of fibrinogenolytic agents and surgical 
intervention are not yet established in pregnancy, though there is undoubtedly an increased tendency to use surgery to prevent extension of thrombi and hence embolization in the postoperative period. Plication of the vena cava may have to be considered, and indeed may be a life-saving procedure, despite the potential long-term effects on the peripheral circulation in the lower limbs. Since pulmonary embolism is a recurrent condition and carries a relatively high mortality, family limitation should be considered in all cases if the mother and child survive. Pregnancy and its management after pulmonary-artery embolectomy has been reported with success. ${ }^{8}$

Finally, it should be emphasized that though antepartum pulmonary embolism is relatively rare it has a high maternal mortality and morbidity. Moreover, there is an increased fetal loss, so the condition should never be taken lightly. Once diagnosed, treatment is required urgently to ensure the best possible outcome.

1 Henderson, S. R., Lund, C. J., and Creasman, W. T., American fournal of Obstetrics and Gynecology, 1972, 112, 476.

Ministry of Health Reports on Confidential Enquiries into Maternal Deaths. London, H.M.S.O., 1957, 1960, 1963, 1966, 1969.

${ }^{3}$ Evans, D. S., and Cockett, F. B., British Me tical fournal, 1969, 2, 802 4 Kakkar, V. V., and Flanc, C., British Fourna! of Surgery, 1968, 55, 384 5 Kakkar, V. V., Howe, C. T., Laws, J. W., an 1 Flanc, C., British Medical Fournal, 1969, 1, 810 .

Kakkar, V. V., Howe, C. T., Flanc, C., and Clarke, M. B., Lancet, 1969 $2,230$.

Jeffcoate, T. N. A., and Tindall, V. R., Australian and New Zealand Fournal of Obstetrics and Gynaecology, 1965, 5, 119.

Tindall, V. R., in Scientific Basis of Obstetrics and Gynaecology, ed. R. R. MacDonald, p. 412. London, Churchill, 1971.

\section{Paris Cancer Symposium}

Immunotherapy was the dominant theme of a cancer symposium held recently in Paris. This was to be expected as it was organized by Professor G. Mathé, who is a pioneer in this field. Though it has been tried for several years, immunotherapy is still at an early stage of its development and may fall into disrepute if used indiscriminately. The evidence that it is effective against some tumours in experimental animals is beyond doubt, but a large gap remains between the experimental results and clinical results in man.

The most widely used form of immunotherapy is nonspecific stimulation of immune responses. B. Benacerraf, in reviewing the action of adjuvants, drew attention to the following key points. The most effective non-specific stimulants of antigens against neoplastic activity are crude preparations of mycobacteria and Corynebacterium parvum. The adjuvants stimulate all types of lymphocytes including both the cytotoxic $T$ lymphocytes and the lymphocytes that enhance the production of antibody. The production of antibody by the B lymphocytes appears to be controlled by $\mathrm{T}$ lymphocytes acting as "helper." But the enhancement of tumoral antibodies may lead to the synthesis of blocking antibodies which protect the tumour cells. It is hoped that the biochemical fractionation of the adjuvants now in progress may yield more specific agents, in particular to enhance the cytotoxic $T$ cells without stimulating the "helper" functions.

The best antitumour effects in animals are obtained when the adjuvant is injected with the tumour cells or at the site of tumour growth. This causes a tuberculin-like reaction in which the infiltrating mononuclear cells kill the cancer cells. However, for any hope of success of cure by immunotherapy animal studies have shown two cardinal rules. The tumour must be antigenically different from the host, and the tumour mass must be very small.

Several investigators reported the use of adjuvants or vaccines of killed tumour cells in the treatment of advanced cancer in man as a last resort or to assess the efficacy of immunotherapy in slowing tumour growth. The growth of some solid tumours may be slowed temporarily, but with a few exceptions the results were negative or conducted on too few patients to permit a scientific evaluation. In acute myeloblastic leukaemia, a disease in which sustained remissions have been rare, the preliminary results of a group in London seem to be encouraging. They reported that repeated injections of irradiated leukaemic cells (derived from other patients) and B.C.G. when combined with chemotherapy are more effective in maintaining patients in remission than chemotherapy alone.

For the immunotherapy of cancer to advance it is essential that the immunological response of the patient to the therapy be measured. The meeting drew attention to the pressing need for better ways of assessing the patient's immunological status and devising tests indicative of immune reactions against cancer cells. Delayed hypersensitivity responses to skin allergens such as tuberculin (P.P.D.), mumps antigens, streptokinase, and Candida seem to vary considerably within groups of patients with comparable tumours. Reassessment of the reaction during the course of the disease is complicated by the fact the patient will be repeatedly exposed to the test allergen, which itself may alter the response. Estimation of the reactivity of blood lymphocytes to phytohaemagglutinin apears to be a reliable index of the numbers of $\mathrm{T}$ lymphocytes among all the circulating lymphocytes. There may be a correlation between reactivity to dinitrochlorobenzene sensitization and prognosis in a variety of cancers, notably in tumours of the head and neck.

If immunotherapy is to be successful against solid tumours, more research is needed into the detection of minimal metatases or early recurrence of a primary growth, for it is at this stage of the disease that immunological treatment would seem to be justified ethically and scientifically.

\section{Mobility of Chest}

It is a truth of clinical medicine that one physical sign which can be measured and given a figure is worth a dozen impressionistic remarks in follow-up notes. Many physicians continue to carry a tape measure to record chest expansion. It is a routine measurement which has to be recorded on most insurance companies' examination forms and is usually a part of a general physical check-up.

In ankylosing spondylitis it is a measure of rigidity in the thoracic wall, for these patients depend mostly on diaphragmatic excursion, the ribs being fused to the transverse processes and bodies of the dorsal vertebrae. The doubleended tape measure with central attachment to fit over the dorsal spine posteriorly was in general use in the 1930s but is rarely seen today. How reliable are such measurements? Is the taking of them justified?

One of the older textbooks on diseases of the chest ${ }^{1}$ states that the difference in circumference between inspiration and expiration in normal man is "about two inches" $(5 \mathrm{~cm})$ and that greater degrees of mobility are found in 